PALABRAS CLAVE

Malnutrición

Niños

Aspectos económicos

Aspectos sociales

Desigualdades regionales

Distribución del ingreso

Análisis de datos

Indicadores económicos

Indicadores de salud

América Latina

Caribe

\section{Desnutrición crónica infantil y desigualdad socioeconómica en América Latina y el Caribe}

\author{
Guillermo Paraje
}

$\mathrm{E}$

n este trabajo se indaga acerca de los factores que determinan el nivel de desnutrición crónica infantil y su distribución socioeconómica en ocho países de América Latina y el Caribe. Para ello se utiliza una metodología que permite descomponer un índice de desigualdad socioeconómica (el índice de concentración) según los factores que lo afectan. En el caso de los países analizados, la "riqueza" de los hogares (medida mediante un indicador de bienestar material) y el nivel educativo de las madres son los determinantes más importantes de la distribución de dicha desnutrición. Los factores biomédicos considerados pueden ser relevantes para explicar el nivel de la desnutrición, pero su contribución para explicar la desigualdad es relativamente baja. Asimismo, factores geográficos, culturales, étnicos e idiosincrásicos juegan un rol explicativo limitado y, aparentemente, lo hacen a partir de su relación con la distribución de las variables socioeconómicas mencionadas. 


\section{I}

\section{Introducción}

La desnutrición crónica infantil tiene enormes implicancias económico-sociales. Por una parte, se relaciona con resultados de salud negativos, especialmente con una mayor mortalidad infantil. Pelletier y otros (1995), por ejemplo, muestran que en promedio el $56 \%$ de las muertes de menores de cinco años en 53 países en desarrollo fueron consecuencia directa o indirecta de deficiencias nutricionales. Más recientemente, Black y otros (2008) reportan que la desnutrición infantil causa alrededor de 2,2 millones de muertes anuales y un $21 \%$ de los años de vida ajustados en función de la discapacidad (DALY) perdidos en países de ingresos medios y bajos.

En general, la desnutrición infantil trae aparejados costos sociales directos — debido a la mayor mortalidad, pero también a la mayor morbilidad y a los costos monetarios que esto implica-e indirectos. Entre estos últimos se pueden mencionar, por ejemplo, una caída permanente en la habilidad cognitiva de los niños, el ingreso tardío al sistema educativo, una mayor deserción escolar, entre otros (Victora y otros, 2008). Estos factores se vinculan a una menor productividad laboral y a un inferior crecimiento económico (OMs, 2001). Dadas estas características, la desnutrición crónica entraña una pérdida permanente de recursos presentes y futuros y una alteración en su distribución.

Por otra parte, la concentración desproporcionada de desnutrición en los estratos socioeconómicos más bajos implicaría, entre otras cosas, que a medida que se incrementa la importancia relativa de los costos económicos directos e indirectos, la desnutrición no solo sería consecuencia de la desigualdad económica, sino que se transformaría en una de sus causas (debido al efecto empobrecedor que tendría entre los afectados), creándose un círculo vicioso.

En el caso de América Latina y el Caribe (ALyC) es notoria esta relación, la que se encuentra expuesta

\footnotetext{
$\square$ El autor agradece los comentarios de Ana Sojo, Andras Uthoff, Ritu Sadana y Ahmad Hosseinpoor, de un árbitro anónimo y de participantes del Seminario internacional "Desigualdad socioeconómica y el derecho a la salud en America Latina y el Caribe", organizado por CEPAL, donde fue presentada una versión anterior de este trabajo. Los errores existentes son de responsabilidad exclusiva del autor.
}

en el gráfico 1, donde se muestra la prevalencia de desnutrición infantil crónica a nivel nacional (porcentaje de niños desnutridos sobre el total) y entre los hogares pertenecientes al quintil más pobre de un gran grupo de países de ingreso medio y bajo. El hecho de que en todos estos países la desnutrición entre los hogares del quintil más pobre sea más frecuente que en un hogar promedio (todas las observaciones se encuentran sobre la línea de $45^{\circ}$ dibujada en el gráfico) denota que esta condición se relaciona invariablemente con la pobreza. Pero en el gráfico se aprecia también que, considerando su prevalencia promedio (baja o alta relativa a otros países en desarrollo), los países de la región tienen prevalencias en el quintil inferior que se encuentran entre las más altas del mundo.

Este hecho tiene implicancias inmediatas en términos de políticas socioeconómicas: si se desea disminuir la desnutrición promedio es necesario reducir su prevalencia entre los hogares más pobres y, por ende, aminorar la desigualdad en su distribución. Entonces, no solo es necesario comprender cuáles son las causas de la desnutrición crónica para actuar sobre ellas, sino que es crucial entender qué variables afectan a su distribución socioeconómica, ya que en principio podrían existir variables que siendo poco importantes para explicar el nivel promedio de la desnutrición sean muy relevantes para explicar su distribución.

El objetivo principal de este trabajo es identificar y cuantificar el efecto que han tenido variables socioeconómicas y biomédicas relevantes no solo en el nivel de la desnutrición crónica infantil, sino en su distribución socioeconómica en ocho países de ALyC durante los primeros años del siglo XXI. Aunque la metodología utilizada no es novedosa y ha sido aplicada en otros casos (Wagstaff, Van Doorslaer y Watanabe, 2003; Hosseinpoor y otros, 2006; Van de Poel y otros, 2007; Chen, Eastwood y Yen, 2007), en este trabajo se realizan diversas contribuciones a la literatura. En primer lugar, se considera a un gran conjunto de países de la región con diferentes niveles de desarrollo y de distintas subregiones (Centroamérica, Caribe, Región Andina). Aunque se conoce que estos países tienen altos niveles de desigualdad económica (De Ferranti y otros, 2004), su efecto en la salud -y especialmente en la desnutrición crónica infantil— ha 
GRÁFICO 1

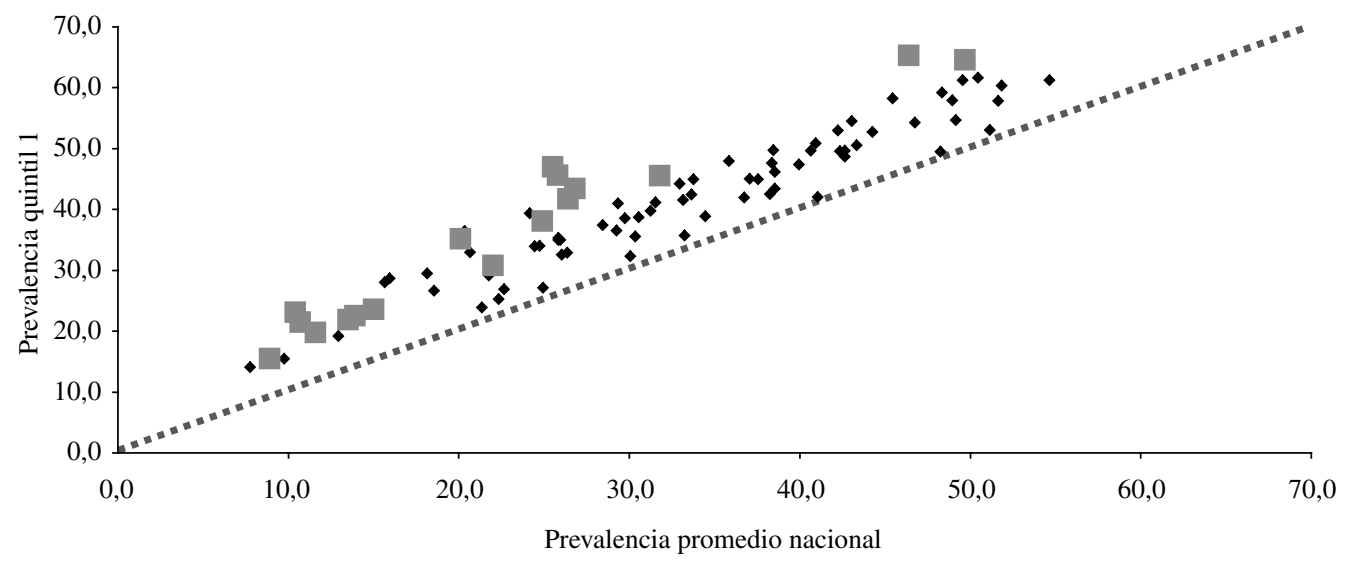

- Países en desarrollo América Latina y el Caribe

Fuente: elaboración del autor sobre la base de D. Gwatkin y otros, Socio-Economic Differences in Health, Nutrition, and Population within Developing Countries. An Overview, Washington, D.C., Banco Mundial, 2007 [en línea] http://go.worldbank.org/XJK7WKSE40.

sido menos investigado, sobre todo en una perspectiva regional. ${ }^{1}$

En segundo lugar, se utiliza el nuevo patrón de referencia de la Organización Mundial de la Salud (OMs) para medir la desnutrición crónica (OMs, 2006). Este nuevo referente, en lugar de considerar como población de comparación la de los Estados Unidos, incluye a grupos poblacionales de países con distintos grados de desarrollo y diferente composición étnica, tales como Brasil, Ghana, India, Noruega y Omán (además de los Estados Unidos). La población de referencia que se toma en ellos es aquella que sigue ciertas conductas recomendables en cuanto a alimentación (principalmente para el recién nacido) y cuidado de la salud. Por ello, es un patrón de referencia más amplio (porque considera diferentes poblaciones) y a la vez más preciso (porque toma grupos específicos dentro de dichas poblaciones) para la medición de las deficiencias nutricionales. Hasta donde el autor conoce, este es el primer trabajo en que se adopta este patrón para el estudio de la desigualdad socioeconómica en la desnutrición crónica infantil en la región. ${ }^{2}$

Finalmente, se utiliza un modelo explicativo simple con variables que tienen un correlato directo con políticas sanitarias, educativas y de ingreso. Estas variables también pueden relacionarse de manera sencilla con modelos propuestos para analizar los determinantes socioeconómicos de la salud, como el recomendado por la reciente Comisión sobre Determinantes Sociales de la Salud (oms, 2008).

La estructura del trabajo es la siguiente. En la sección II se presenta la metodología utilizada para descomponer los factores determinantes que repercuten en la desnutrición crónica, como también en su distribución socioeconómica. Además, se describen las fuentes de datos utilizadas en el estudio. En la sección III se presentan los resultados de los análisis realizados, mientras que en la sección IV se entregan las conclusiones del estudio y se presenta una serie de recomendaciones de política destinadas a reducir el impacto de la desnutrición crónica y su desigual distribución socioeconómica.
${ }^{1}$ Solo se han encontrado tres estudios donde se comparan diferencias socioeconómicas para diferentes grupos de países de ALyC (Larrea, 2002; Larrea y Freire, 2002; Martínez, 2005). Sin embargo, estos autores utilizan una metodología distinta a la que aquí se sigue.

\footnotetext{
2 En Paraje (2008) y CEPAL (2008) se utiliza la misma metodología que en este trabajo, pero la desnutrición infantil se mide utilizando el patrón de referencia antiguo (basado solo en la población de los Estados Unidos).
} 


\section{II}

\section{Metodología y fuente de datos}

Existe una serie de factores que, a nivel agregado, pueden relacionarse con la desnutrición crónica, cuya causa primaria es la insuficiente ingesta y asimilación de nutrientes. Estos factores podrían agruparse en al menos cuatro categorías (Martínez y Fernández, 2006). Primero, los factores medioambientales - como fenómenos naturales (inundaciones, terremotos, sequías, y otros) - y "entrópicos" (vale decir, causados por la acción humana sobre el medio ambiente), como la contaminación ambiental, y que pueden afectar temporal o definitivamente la posibilidad de producir alimentos o de generar ingresos a las familias que los sufren.

Pero incluso si estos factores no se encuentran presentes o su influencia es moderada, la producción de alimentos o la generación de ingresos puede ser abundante a nivel agregado, pero insuficiente a nivel individual debido a la distribución desigual de los derechos (entitlements) para acceder a ellos. ${ }^{3}$ Segundo, los factores llamados socioeconómico-culturales pueden determinar la asignación de esos derechos mediante la distribución de los activos productivos (capital físico y humano) y, en consecuencia, de los ingresos.

En tercer lugar, se cuentan los factores productivos que incluyen las "características de los procesos productivos", el "nivel de aprovechamiento que estos hagan de los recursos naturales" y "el grado en que dichos procesos mitigan o aumentan los riesgos medioambientales" (Martínez y Fernández, 2006, p. 33).

Finalmente, en los factores biomédicos se incluyen elementos que pueden incidir en la propensión individual a adquirir desnutrición, como por ejemplo, el estado nutricional materno (sobre todo durante la gestación y los primeros meses de vida del niño), la duración de la lactancia materna (un menor período de lactancia tiende a aumentar la probabilidad de desnutrición infantil), el sexo y la edad del niño, los factores congénitos, y otros.

En la metodología aplicada en este trabajo para aproximarse al problema de la desnutrición crónica infantil desde una perspectiva cuantitativa, se considera de manera directa e indirecta a este conjunto de factores. Esta metodología consiste en, primero, estimar una

\footnotetext{
${ }^{3}$ Este argumento es claramente expuesto por Drèze y Sen (1989) para explicar cómo pueden producirse hambrunas incluso en circunstancias de relativa abundancia general de alimentos.
}

regresión multivariada entre la variable de desnutrición crónica infantil y un conjunto de variables independientes relevantes, y luego sobre la base de dicha estimación descomponer la desigualdad socioeconómica de la desnutrición en sus factores causantes.

En gran parte de la literatura sobre este tema, se supone que la relación estadística a tener en cuenta en el primer paso de este proceso es la forma reducida de una función de producción de desnutrición crónica infantil en el interior de los hogares (Grossman, 1972), que se estima a nivel país. Mediante esta función se consideran todos los factores que influyen directamente en el nivel de desnutrición crónica infantil promedio, así como variables no incluidas directamente en la estimación, pero cuya influencia en la desnutrición se intermedia a partir de las variables incluidas. Por ejemplo, puede considerarse el grupo étnico al que pertenece un niño como un factor decisivo en su grado de desnutrición infantil. Si esta influencia ocurre porque, por ejemplo, ese grupo étnico posee características genéticas o factores no observables que lo diferencian de los otros grupos y que son los que causan la desnutrición, entonces la pertenencia o no a esta etnia debería ser considerada como una variable relevante dentro de esta "función de producción de desnutrición crónica infantil". ${ }^{4} \mathrm{Si}$, por el contrario, este grupo étnico posee altos niveles de desnutrición (más elevados que el promedio) y se considera que estos podrían ser causados por bajos niveles de ingreso (menores al promedio) o niveles insuficientes de educación de los padres, entonces son estas variables (ingresos, educación, y otras) las que debieran considerarse dentro de la explicación y no las variables étnicas propiamente tales.

En el presente trabajo, la función propuesta a estimar tiene la siguiente forma lineal:

$$
z_{i, t}=\beta^{0}{ }_{t}+\Sigma \beta^{k}{ }_{t} x_{i, t}+\Sigma \beta^{m}{ }_{t} x^{m}{ }_{h, t}+\varepsilon_{i, t}
$$

donde $z_{i, t}$ es el nivel nutricional del individuo $i$ en el país $t ; x_{i, t}^{k}$ es un conjunto de variables explicativas a nivel

\footnotetext{
${ }^{4} \mathrm{El}$ efecto de las variables étnicas en la desnutrición ha sido considerado como irrelevante en el estudio multicéntrico de la Organización Mundial de la Salud (oms) para determinar patrones de referencia de crecimiento en la infancia (OMs, 2006).
} 
de cada individuo, $i$; $x^{m}{ }_{h, t}$ es un conjunto de variables explicativas a nivel de cada hogar, $h$. Los coeficientes que acompañan a estas variables son, entonces, una estimación estadística de la importancia marginal de cada uno de estos factores a la hora de explicar el nivel de desnutrición promedio.

En una segunda etapa y a partir de la estimación de esta relación lineal, es posible utilizar ciertos indicadores de desigualdad socioeconómica del estado nutricional de los niños y descomponer en sus causas esa desigualdad. El indicador elegido para tal efecto es el índice de concentración, cuya interpretación (y cálculo) es similar a la del conocido coeficiente de Gini (utilizado para medir desigualdad en el ingreso) $y$, como este, es derivado de una herramienta gráfica de fácil interpretación: la curva de concentración (homóloga a la curva de Lorenz). ${ }^{5}$ En el caso de la desnutrición crónica, esta curva grafica el estado nutricional (deficiencia nutricional) acumulado de los niños, de acuerdo con la posición socioeconómica

5 Para una descripción de este índice y de sus propiedades asintóticas, véanse Kakwani, Wagstaff y Van Doorslaer (1997). (por ejemplo, medida por ingreso o riqueza) de sus hogares. El índice de concentración es igual a dos veces el área comprendida entre la curva de concentración y la línea de $45^{\circ}$ (que marca una distribución neutra de la variable considerada).

Si la curva de concentración se halla por debajo de la diagonal, el índice de concentración toma valores en el rango $[0 ; 1]$ (indicando que la variable analizada se concentra entre los hogares de mayor nivel socioeconómico). En caso contrario, toma valores en el rango $[-1 ; 0]$. Cuanto más (menos) concentrada se encuentre esta variable más (menos) cerca estará este índice del valor uno (en valor absoluto). En el caso de la desnutrición crónica infantil, es altamente probable que el índice esté entre $[-1 ; 0]$, mostrando una concentración en los hogares pobres. En el gráfico 2 se muestra la curva de concentración de la desnutrición infantil para Honduras (2005), con un índice de concentración igual a $-0,191$.

De acuerdo con Wagstaff, Van Doorslaer y Watanabe (2003), si la desnutrición crónica puede ser explicada por la ecuación (1), la desigualdad socioeconómica en esa desnutrición, resumida en su índice de concentración, puede ser descompuesta de la siguiente forma:

GRÁFICO 2

Honduras: curva de concentración de la desnutrición crónica infantil, 2005 (En porcentajes)

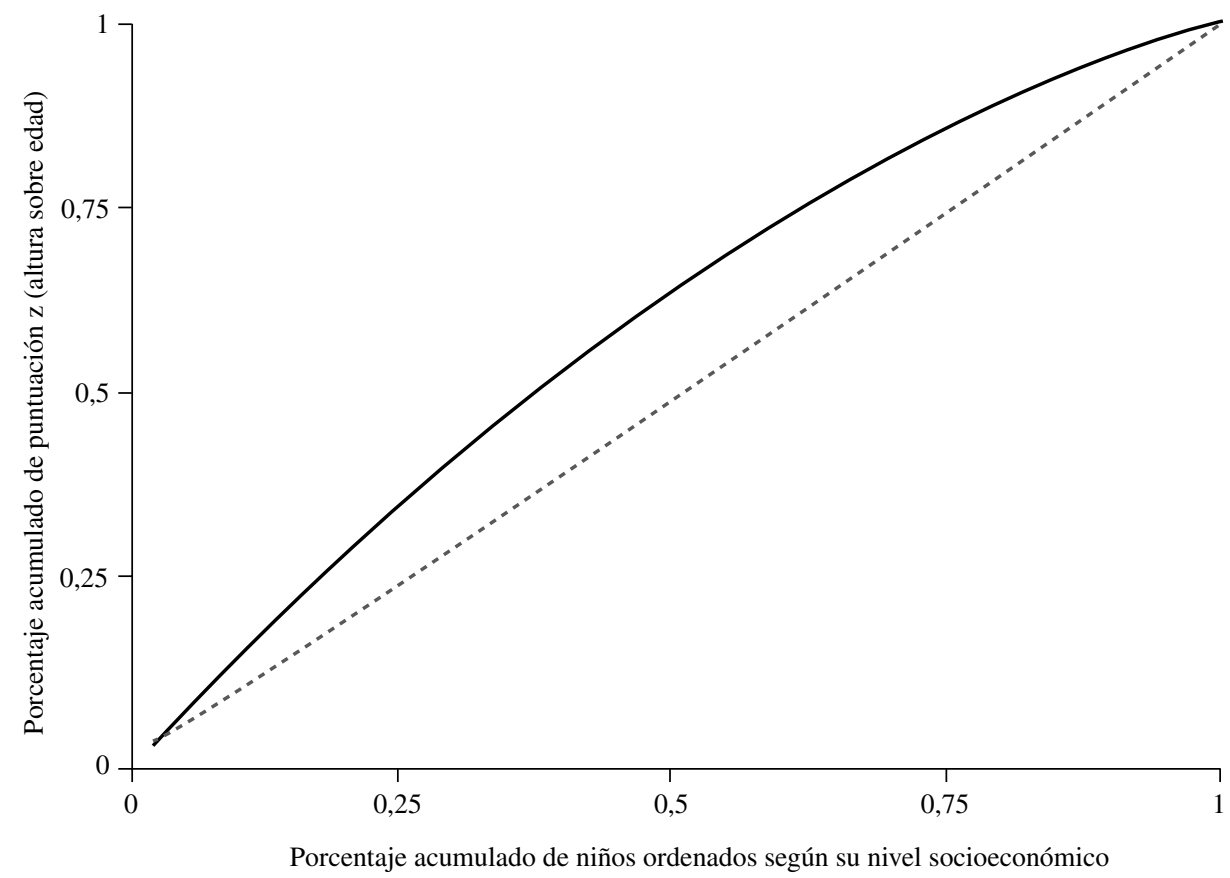

Fuente: elaboración propia sobre la base de las encuestas de demografía y salud (DHS). 


$$
\begin{aligned}
& C I_{t}^{z}=\sum\left(\beta_{t}^{k} \bar{x}_{t}^{k} / \bar{z}_{t}\right) C I_{t}^{k}+ \\
& \sum\left(\beta_{t}^{m} \bar{x}_{t}^{m} / \bar{z}_{t}\right) C I_{t}^{m}+\left(G_{\varepsilon} / \bar{z}_{t}\right)
\end{aligned}
$$

donde $\mathrm{CI}_{t}$ es el índice de concentración de la desnutrición crónica para el país $t ; \mathrm{CI}_{t}{ }_{t}$ y $\mathrm{CI}^{m}{ }_{t}$ son los índices de concentración de las variables explicativas; las expresiones $\left(\beta_{t} \bar{x}_{t} / \bar{z}_{t}\right)$ son las "elasticidades" de la desnutrición crónica respecto de las variables explicativas; y $\left(G_{\varepsilon} / \bar{z}_{t}\right)$ es un término residual que recoge todos los elementos no explicados por la relación (1). ${ }^{6}$

Finalmente, dividiendo ambos términos de (2) por $\mathrm{CI}^{z}{ }_{t}$ se obtiene la importancia relativa de cada variable sobre la desigualdad socioeconómica de la variable explicada:

$$
\begin{aligned}
& 1=\sum\left(\beta_{t}^{k} \bar{x}_{t}^{k} / \bar{z}_{t}\right)\left(C I_{t}^{k} / C I_{t}^{z}\right)+\sum\left(\beta_{t}^{m} \bar{x}_{t}^{m} / \bar{z}_{t}\right) \\
& \left(C I_{t}^{m} / C I_{t}^{z}\right)+\left[\left(G_{\varepsilon} / \bar{z}_{t}\right) / C I_{t}^{z}\right]
\end{aligned}
$$

En la ecuación (3) se muestra la contribución de cada variable a la desigualdad socioeconómica total en la desnutrición infantil (lado izquierdo de la ecuación), ponderada por su importancia a la hora de explicar el nivel promedio de la desnutrición.

Tres elementos afectan a dicha contribución. El primero es la importancia que tiene este factor con respecto al nivel promedio de desnutrición crónica y está dado por el coeficiente de cada variable en las regresiones lineales reportadas (coeficientes $\square$ ). Si este tiene un valor alto, el factor va a tener un mayor "peso" explicativo en la desigualdad de la desnutrición. El segundo elemento es el nivel medio que alcanza dicha variable, en relación con el nivel medio de la desnutrición crónica. Cuanto mayor sea la media de una variable, mayor va a ser su contribución relativa a la desigualdad de la desnutrición. Estos dos elementos (coeficiente de la regresión y media de la variable) sirven para estimar la "elasticidad" de cada variable con respecto a la desnutrición crónica (y se utilizan como ponderador de la influencia de la desigualdad

\footnotetext{
${ }^{6}$ En el apéndice de Wagstaff, Van Doorslaer y Watanabe (2003) se detalla cómo se obtiene la ecuación (2), pero es un resultado bien conocido de la literatura de la distribución del ingreso. El término de residuo puede ser interpretado como el índice de concentración del error de la regresión (1), $\square$. Un residuo nulo o pequeño implicaría entonces que el error de la regresión no tiene ninguna distribución específica con respecto a la variable socioeconómica utilizada para estratificar a los hogares.
}

socioeconómica de cada variable explicativa en la desigualdad de la desnutrición infantil). Finalmente, el tercer elemento es la concentración socioeconómica de cada variable o, en otras palabras, su índice de concentración. En la medida que una variable tenga un impacto importante sobre el resultado (esto es, alta elasticidad sobre la desnutrición) y se encuentre relativamente concentrada en un grupo específico (por ejemplo, hogares "pobres"), esa variable va a contribuir de mayor manera a explicar la concentración relativa del resultado.

Los datos utilizados para estimar las ecuaciones (1), (2) y (3) provienen de las encuestas de demografía y salud (DHs, por su sigla en inglés), recopiladas por Macro International Inc. y disponibles en www. measuredhs.com, que se realizan en más de 75 países de ingreso medio-bajo e ingreso bajo en diferentes regiones del mundo. Son encuestas de hogares, con representatividad nacional y subnacional y un tamaño muestral importante (usualmente entre 5.000 y 30.000 hogares), cuyo objetivo principal es medir condiciones de vida, conductas sanitarias y resultados de salud de mujeres en edad reproductiva y niños. Los indicadores así recopilados tienen la ventaja de ser totalmente comparables en el interior de los países a lo largo del tiempo y entre países. Son recopiladas a intervalos no regulares, normalmente cada cinco años. Las DHS utilizadas en este caso son las últimas disponibles (en el momento de realizarse este trabajo) para los siguientes países (el año de la encuesta se señala entre paréntesis):

1. Bolivia (2003)

2. Colombia (2005)

3. República Dominicana (2007)

4. Haití (2005)

5. Honduras (2005)

6. Guatemala (1999)

7. Nicaragua (2001)

8. Perú (2004)

La variable dependiente a estimar en la ecuación (1) es una medida frecuentemente utilizada como indicador del estado nutricional crónico o de largo plazo del niño: el coeficiente estandarizado de altura sobre edad (también llamado puntuación z). Usualmente, si este coeficiente se encuentra por debajo de las dos desviaciones estándar de la mediana de la población de referencia, se considera al niño como desnutrido crónico (es decir, un niño con deficiencias nutricionales sostenidas en el tiempo, con consecuencias en su crecimiento corporal normal). En todos los países, la población considerada corresponde a niños menores de cinco años (hasta 59 meses de vida, 
incluidos), excepto en el caso de Bolivia, que registra datos solo para niños menores de 36 meses de vida.

En este trabajo no se utiliza una variable dicotómica para medir desnutrición o ausencia de desnutrición, sino que se usa la misma puntuación z (o, mejor dicho, su negativo para facilitar la interpretación de los resultados). De esta manera, es posible capturar toda la información referida a la situación nutricional (estado nutricional y, en caso de que exista, profundidad de la desnutrición), contrariamente a lo que sucedería si se utilizara una variable dicotómica (en ese caso, solo se mediría la existencia o inexistencia de desnutrición en el niño).

Las variables explicativas de la ecuación (1) han sido seleccionadas en función de la evidencia teóricoempírica aportada en la literatura económica y de la disponibilidad de información en la fuente seleccionada. En este sentido, las relaciones estimadas entre desnutrición crónica infantil y el conjunto de variables explicativas constituyen estimaciones de "formas reducidas", que incorporan implícitamente cierto número de relaciones entre la desnutrición y variables no utilizadas directamente. Variables relevantes no medidas por las DHS, como la cultura o las diferencias idiosincrásicas entre grupos, son consideradas de manera colateral mediante la región del país o las variables educativas de los padres. Tal como se mencionó anteriormente, que estas variables no aparezcan explícitamente en las relaciones estimadas no implica desconocer su importancia relativa, sino suponer que actúan indirectamente a partir de su influencia en otros canales más directos.

Las variables explicativas utilizadas pueden agruparse, siguiendo el esquema de Martínez y Fernández (2006), en factores socioeconómicos, factores ambientales y factores biomédicos. ${ }^{7}$ Entre los primeros, medidos a nivel del hogar, se encuentra el índice de "riqueza" o de "bienestar material". Este índice se construye empleando análisis de componentes principales (metodología descrita en Filmer y Pritchett, 2001), en que se usa la información existente acerca de las condiciones materiales de los hogares (disponibilidad de electricidad, personas por habitación, material de pisos, paredes y techos, entre otras) y la posesión de ciertos activos (automóvil, motocicleta, bicicleta, televisor, refrigerador, y otros). En

\footnotetext{
${ }^{7}$ No existe una correspondencia directa entre las variables utilizadas en este trabajo y las propuestas por Martínez y Fernández (2006), debido principalmente a la ausencia de datos para algunas de ellas. Por ejemplo, mediante las variables ambientales empleadas aquí (que son, en realidad, variables geográficas) se procura capturar algunas de las causas propuestas en dicho trabajo.
}

las DHS no se recopila ningún tipo de variable monetaria (como ingresos o gastos) y el indicador de "riqueza" es lo único que puede utilizarse para medir el bienestar material de los hogares. ${ }^{8}$ Es esperable que a mayor valor de este índice (mayor "riqueza" del hogar), menor sea la desnutrición crónica infantil, debido a las mayores oportunidades que tendrían los hogares de asegurar una fuente estable de alimentación para los niños. ${ }^{9}$

Además, se utilizan la educación formal materna medida en años de escolarización completados; la educación formal del esposo o pareja medida también en años de escolarización completados (en el caso de mujeres solteras o sin pareja se considera el nivel educativo del jefe de hogar); y la situación laboral de la madre (empleo calificado versus empleo no calificado, siendo la no inserción en el mercado laboral el grupo de referencia). Es de esperar que las variables educativas también tengan una influencia negativa sobre la desnutrición crónica (y sobre los resultados de salud en general, como muestran Armar-Klemesu y otros, 2000; Harttgen y Misselhorn, 2006), especialmente en el caso de la educación materna. Los canales por los que esta variable actúa son a menudo variados y complejos y pueden incluir prácticas de higiene adecuadas, mayor capacidad para aprender mejores prácticas de cuidado infantil, mejor uso de los servicios sanitarios públicos o privados, y otras (Cleland y Van Ginneken, 1998). Asimismo, la mayor educación materna es a menudo fuente de mayores ingresos para el hogar, lo que refuerza su influencia positiva. Debido a la posible existencia de efectos no lineales, la variable de educación materna también es considerada al cuadrado (como en Larrea y Kawachi, 2005). Un coeficiente positivo (negativo) para esta variable mostraría retornos marginales crecientes (decrecientes) de la educación materna sobre la desnutrición.

Respecto de las variables laborales existen al menos dos efectos contrapuestos que podrían afectar a la situación nutricional del niño. Por una parte, que la madre trabaje fuera del hogar implica mayores ingresos y, por ende, una mejor situación socioeconómica (y una menor desnutrición infantil). Por otra, el trabajo materno fuera del hogar implica que el niño debe quedar a cargo de

\footnotetext{
${ }^{8}$ Para una descripción de las ventajas y limitaciones de este indicador, véanse Rutstein y Johnson (2004) y Paraje (2008).

${ }^{9}$ En numerosos estudios se ha encontrado una estrecha relación entre indicadores de bienestar material (ya sea el índice de "riqueza" utilizado aquí o ingresos/gastos del hogar provenientes de otras encuestas) y la desnutrición crónica infantil, entre ellos Wagstaff, Van Doorslaer y Watanabe (2003); Harttgen y Misselhorn (2006); Van de Poel y otros (2007); Chen, Eastwood y Yen (2007).
} 
terceros que no necesariamente tienen las mismas calificaciones que la madre, lo que afecta (negativamente) la salud del niño. ${ }^{10}$ Es probable que para trabajos de baja calificación este efecto negativo sobrepase al efecto positivo mencionado, siendo el trabajo materno, finalmente, una causa de la desnutrición infantil (manteniendo el resto de los factores constantes). Larrea y Kawachi (2005) consideran estos canales para el caso del Ecuador y encuentran una relación negativa (no significativa) entre desnutrición y niños no cuidados por sus padres. Lamentablemente, esta última variable no existe en las DHS como para incluirla en este trabajo.

Los factores ambientales (a nivel del hogar) considerados incluyen el área donde se encuentra el hogar (urbana versus rural) y la región político-geográfica. En general, en la literatura se documenta que el sector urbano posee mejores indicadores sanitarios que el sector rural, sobre todo en lo atinente a desnutrición infantil (Smith, Ruel y Ndiaye, 2005). Sin embargo, estas diferencias suelen ocultar grandes disparidades en el interior de dichos grupos, causadas - entre otras cosas - por la distribución de características socioeconómicas, tales como ingreso, educación, composición de los hogares, y otras. Cuando estas características son tenidas en cuenta, las disparidades en resultados de salud entre hogares urbanos y rurales desaparecen o se reducen notablemente (Van de Poel y otros, 2007).

La región político-geográfica también puede determinar los resultados sanitarios. En países donde, por ejemplo, existe concentración geográfica de grupos étnicos, de ciertas actividades económicas o de cobertura de servicios de atención de salud, la incidencia de la desnutrición crónica infantil puede mostrar diferencias importantes en el plano regional (Martínez, 2005; Chen, Eastwood y Yen, 2007). Para los fines de este trabajo, las regiones y distritos subnacionales han sido agrupados según lo propuesto por las DHs. Una definición de estos grupos se encuentra en el cuadro A del Anexo.

En los factores biomédicos (a nivel de cada niño) se incluyen el sexo (el grupo de referencia son los niños varones), la edad en meses en el momento de la encuesta, la edad al cuadrado (para considerar relaciones no-lineales entre esta variable y la desnutrición infantil), su orden de nacimiento (el grupo de referencia es primer hijo) y su peso en gramos al nacer. ${ }^{11} \mathrm{El}$ sexo se utiliza para

\footnotetext{
${ }^{10}$ De hecho, es muy factible que la persona que queda a cargo del niño cuando la madre trabaja fuera del hogar tenga menor calificación laboral que la madre y, muy probablemente, menos educación.

${ }^{11} \mathrm{El}$ peso del niño al nacer es una variable con muchos valores no reportados. Para evitar perder esta importante variable se realizó una
}

considerar la posible existencia de un sesgo de género. Por lo general, existe poca evidencia de que este sea el caso y cuando estas diferencias se encuentran, tienden a mostrar que los niños varones suelen ser más afectados por la desnutrición que las niñas (Marcoux, 2002; Chen, Eastwood y Yen, 2007).

La edad suele estar positivamente relacionada con la desnutrición, pero de manera no lineal: la desnutrición crece bruscamente durante los primeros meses de vida y se estabiliza más adelante (Valdivia, 2004; Larrea y Kawachi, 2005; Chen, Eastwood y Yen, 2007). Por ejemplo, en el caso de los niños de Bolivia, Colombia, el Ecuador y el Perú este punto de estabilización ocurre cerca del vigésimo mes de vida (Martínez, 2005; Valdivia, 2004).

El orden de nacimiento también puede relacionarse con la desnutrición crónica. Conforme este aumenta, se incrementa la probabilidad de que el niño sea desnutrido, debido a que niños de mayor orden deben "competir" con sus hermanos de más edad en cuidado materno, alimentación, entre otros (Martínez, 2005). Finalmente, el peso al nacer se relaciona estrechamente con la morbilidad en los primeros meses de vida, la capacidad de absorber nutrientes de manera adecuada y, en general, el desarrollo futuro (Jewell, Triunfo y Aguirre, 2007; Victora y otros, 2008). Este indicador es más directo y tiene un mayor impacto en la desnutrición que, por ejemplo, el índice de masa corporal de la madre (utilizado por Smith, Ruel y Ndiaye, 2005; Harttgen y Misselhorn, 2006), y por ello es preferido.

Finalmente, se considera una variable que mide el acceso de los hogares al sistema de salud. Es esperable que, manteniendo el resto de las variables constantes $\mathrm{y}$ un sistema de salud que funcione adecuadamente brindando servicios de calidad a un bajo precio, el acceso a esos servicios reduzca la desnutrición infantil. Lamentablemente no existe en las DHs una variable que mida esta dimensión y que pueda vincularse teóricamente al problema de la desnutrición. Una de las alternativas disponibles es utilizar cuidados prenatales (con cuidados apropiados sería posible detectar, por ejemplo, factores congénitos que podrían causar un bajo peso al nacer o se podría mejorar la nutrición materna). Sin embargo,

imputación utilizando el método hot-deck con el tamaño del niño al nacer reportado por la madre (muy grande, más grande que el promedio, promedio, menor al promedio, muy pequeño) y la educación de la madre (sin educación, primaria incompleta/completa, secundaria incompleta/completa, terciaria). Debido a que la educación materna se considera usando categorías, la variable resultante no presenta problemas de colinealidad con la de educación materna en años. 
tales cuidados requerirían de al menos cuatro visitas a profesionales calificados, que aun en el caso de que no cobraran por sus servicios, implicaría un costo económico para la madre (por ejemplo, en términos de tiempo, transporte, y otros). La decisión de tener o no el cuidado adecuado pasaría entonces a depender de la magnitud relativa de dicho costo y, en definitiva, de la situación socioeconómica de la madre. Incluir una variable como esta significaría, en términos estadísticos, tener un problema de endogeneidad (Schultz, 1984).

Para evitar esto es que se elige una variable alternativa: la atención profesional en el parto (atención profesional comparada con no profesional). ${ }^{12} \mathrm{Si}$ bien esta variable no se relaciona estrictamente con el resultado nutricional inmediato del niño, puede afectarlo ya que la atención profesional en el parto puede reducir la morbilidad infantil y materna postparto y ayudar a la madre a obtener información acerca de cómo cuidar mejor al niño (Smith, Ruel y Ndiaye, 2005). Esta variable se incluye entonces no solo por tener esta consecuencia sobre la desnutrición, sino fundamentalmente para medir la disponibilidad (acceso) a servicios básicos de salud (cuidado de la salud, vacunaciones y otros). Implícitamente se asume que si la madre tiene acceso al sistema de salud en el momento del parto, también posee dicho acceso en circunstancias menos críticas.

A manera informativa, en el cuadro 1 se exponen porcentajes de prevalencia de la desnutrición crónica infantil (porcentaje de niños desnutridos sobre el total de niños) cuando se divide la población en grupos basados en las variables explicativas propuestas.

En todos los casos, la desnutrición crónica es más alta en los hogares rurales que en los urbanos y no es infrecuente encontrar prevalencias dos (por ejemplo, Colombia, Haití y Nicaragua) y hasta cuatro veces superiores (Perú) entre los primeros en comparación con los segundos. Además, la desnutrición se incrementa conforme se reduce el quintil de "riqueza" a la que pertenece

12 Se considera que un parto ha sido atendido por un profesional cuando al frente de este ha estado un médico profesional, una enfermera o "matrona" profesional, una "matrona" auxiliar o algún otro profesional (definido por cada país). el hogar y cuanto menor sea la educación materna o de la pareja. Por su parte, la relación entre desnutrición y situación laboral de la madre es menos clara. En algunos países (por ejemplo, Colombia, Guatemala, Nicaragua y República Dominicana) las mayores tasas de desnutrición crónica se verifican entre niños cuyas madres no tienen trabajos remunerados. Pero en otros (Bolivia, Haití, Perú) la mayor prevalencia se encuentra entre niños cuyas madres poseen trabajos no calificados.

En cuanto a las variables biomédicas, en todos los casos los niños varones poseen una mayor prevalencia que las mujeres y esta crece a medida que aumenta el orden de nacimiento (principalmente entre los niños que son terceros o posteriores) y la edad (es muy superior entre niños mayores de un año que entre los menores de un año). Finalmente, en todos los países la prevalencia de desnutrición es considerablemente inferior entre niños cuyos partos fueron realizados por profesionales que entre los que no tuvieron esa atención.

Debe tenerse en cuenta que en los porcentajes del cuadro 1 no se consideran las diferentes interacciones entre las variables y que serán consideradas en el análisis multivariado a continuación.

En el cuadro 2 se presentan estadísticas descriptivas (media y errores estándar robustos) de todas las variables utilizadas. En la primera línea se aprecia, por ejemplo, la media de la puntuación z de la desnutrición crónica. Por la transformación mencionada anteriormente (que consiste en multiplicar por -1 la puntuación z original), los países con mayor media de este indicador son aquellos donde el estado nutricional promedio de los niños es más deficiente. En este sentido, países como Guatemala, Perú, Honduras y Bolivia presentan un peor estado nutricional promedio que Colombia y República Dominicana.

Entre las variables explicativas elegidas, la educación materna - que como se señala en la sección siguiente es uno de los principales determinantes de la desnutrición crónica infantil- también muestra discrepancias importantes entre los países analizados. Por ejemplo, en República Dominicana las madres de la muestra poseen nueve años de escolarización promedio, mientras que en Guatemala esa variable alcanza a solamente 3,3 años. 
CUADRO 1

América Latina y el Caribe (países seleccionados):

prevalencia de la desnutrición crónica según factores determinantes, 1999-2007

(En porcentajes)

\begin{tabular}{|c|c|c|c|c|c|c|c|c|}
\hline Variables & $\begin{array}{c}\text { Bolivia } \\
2003\end{array}$ & $\begin{array}{l}\text { Colombia } \\
2005\end{array}$ & $\begin{array}{c}\text { República } \\
\text { Dominicana } \\
2007\end{array}$ & $\begin{array}{c}\text { Guatemala } \\
2002\end{array}$ & $\begin{array}{l}\text { Haití } \\
2005\end{array}$ & $\begin{array}{c}\text { Honduras } \\
2005\end{array}$ & $\begin{array}{c}\text { Nicaragua } \\
2001\end{array}$ & $\begin{array}{l}\text { Perú } \\
2004\end{array}$ \\
\hline Promedio nacional & 30,9 & 15,6 & 9,8 & 52,5 & 27,6 & 29,3 & 24,4 & 29,9 \\
\hline \multicolumn{9}{|l|}{ Regiones } \\
\hline Región 0 & 34,0 & 16,4 & 7,4 & 32,7 & 19,9 & 18,6 & 8,7 & 8,7 \\
\hline Región 1 & 40,1 & 18,1 & 8,2 & 63,9 & 31,5 & 23,4 & 20,1 & 22,9 \\
\hline Región 2 & 31,3 & 14,5 & 11,5 & 55,4 & 31,6 & 27,5 & 33,6 & 42,2 \\
\hline Región 3 & 41,9 & 13,3 & 9,0 & 52,2 & - & 35,4 & 32,6 & 32,4 \\
\hline Región 4 & 46,6 & 15,8 & 9,0 & 54,6 & - & 47,1 & - & - \\
\hline Región 5 & 22,2 & - & 15,5 & 61,7 & - & 17,9 & - & - \\
\hline Región 6 & 16,6 & - & 8,9 & 74,2 & - & 30,0 & - & - \\
\hline Región 7 & 36,5 & - & 17,4 & - & - & 32,6 & - & - \\
\hline Región 8 & 30,2 & - & 12,0 & - & - & 53,6 & - & - \\
\hline Región 9 & - & - & 11,6 & - & - & 50,8 & - & - \\
\hline Región 10 & - & - & - & - & - & 55,2 & - & - \\
\hline Región 11 & - & - & - & - & - & 48,6 & - & - \\
\hline Región 12 & - & - & - & - & - & 27,9 & - & - \\
\hline Región 13 & - & - & - & - & - & 32,9 & - & - \\
\hline Región 14 & - & - & - & - & - & 23,6 & - & - \\
\hline Región 15 & - & - & - & - & - & 24,9 & - & - \\
\hline \multicolumn{9}{|l|}{ Area } \\
\hline Rural & 40,3 & 22,1 & 12,7 & 61,5 & 32,3 & 38,0 & 34,7 & 47,4 \\
\hline Urbana & 24,0 & 12,5 & 8,4 & 37,2 & 18,5 & 17,2 & 14,2 & 13,2 \\
\hline \multicolumn{9}{|l|}{ "Riqueza" } \\
\hline Quintil 1 & 46,2 & 25,3 & 16,5 & 72,8 & 37,7 & 49,8 & 41,1 & 57,0 \\
\hline Quintil 2 & 38,6 & 16,4 & 9,8 & 67,4 & 35,8 & 38,5 & 31,3 & 40,8 \\
\hline Quintil 3 & 25,2 & 13,5 & 7,2 & 60,2 & 31,5 & 24,9 & 20,5 & 18,7 \\
\hline Quintil 4 & 19,5 & 9,8 & 7,7 & 33,6 & 19,9 & 14,3 & 10,3 & 7,3 \\
\hline Quintil 5 & 12,9 & 4,9 & 4,5 & 12,2 & 6,2 & 7,2 & 5,7 & 6,0 \\
\hline \multicolumn{9}{|l|}{ Educación de la madre } \\
\hline Sin educación & 48,6 & 31,6 & 16,2 & 70,8 & 37,9 & 52,6 & 41,3 & 61,1 \\
\hline Primaria incompleta/completa & 37,7 & 19,9 & 12,6 & 49,1 & 26,8 & 31,4 & 25,7 & 47,6 \\
\hline Secundaria incompleta/completa & 18,8 & 11,6 & 9,2 & 19,3 & 14,2 & 13,8 & 10,6 & 17,5 \\
\hline Terciaria incompleta/completa & 13,8 & 5,7 & 4,7 & 3,3 & 2,6 & 5,4 & 6,6 & 5,5 \\
\hline \multicolumn{9}{|l|}{ Situación laboral de la madre } \\
\hline No trabaja fuera del hogar & 27,3 & 20,1 & 10,3 & 54,8 & 25,9 & 31,9 & 26,8 & 20,2 \\
\hline Trabajo calificado & 28,0 & 6,0 & 6,5 & 41,0 & 9,0 & 21,6 & 13,0 & 10,8 \\
\hline Trabajo no calificado & 34,0 & 15,7 & 10,6 & 49,4 & 30,4 & 26,7 & 23,3 & 37,8 \\
\hline \multicolumn{9}{|l|}{ Educación de la pareja } \\
\hline Sin educación & 43,1 & 21,5 & 16,9 & 66,3 & 37,2 & 47,7 & 36,5 & 57,3 \\
\hline Primaria incompleta/completa & 40,3 & 19,5 & 12,5 & 57,6 & 32,0 & 30,6 & 27,3 & 47,8 \\
\hline Secundaria incompleta/completa & 22,7 & 11,3 & 8,3 & 22,9 & 16,4 & 15,4 & 27,1 & 24,5 \\
\hline Terciaria incompleta/completa & 14,0 & 6,1 & 7,1 & 15,5 & 8,8 & 8,8 & 7,2 & 9,7 \\
\hline \multicolumn{9}{|l|}{ Sexo } \\
\hline Masculino & 34,0 & 16,9 & 11,2 & 54,0 & 30,2 & 31,3 & 25,7 & 34,4 \\
\hline Femenino & 27,6 & 14,3 & 8,3 & 50,8 & 25,2 & 27,3 & 23,1 & 25,3 \\
\hline \multicolumn{9}{|l|}{ Orden de nacimiento } \\
\hline Primer niño & 22,2 & 11,1 & 7,9 & 42,4 & 22,1 & 20,6 & 18,5 & 20,8 \\
\hline Segundo niño & 28,1 & 15,8 & 8,2 & 41,8 & 20,9 & 24,7 & 20,3 & 22,1 \\
\hline Tercer niño & 30,1 & 16,0 & 10,9 & 49,7 & 24,6 & 28,0 & 20,9 & 27,4 \\
\hline Cuarto o posterior & 38,4 & 23,5 & 14,2 & 63,8 & 35,7 & 40,8 & 34,4 & 48,9 \\
\hline \multicolumn{9}{|l|}{ Edad } \\
\hline Menor de 12 meses & 14,6 & 9,3 & 8,2 & 30,5 & 14,7 & 13,2 & 9,6 & 12,9 \\
\hline Entre 12 y 24 meses & 35,2 & 18,5 & 12,7 & 55,2 & 31,6 & 28,9 & 24,5 & 32,9 \\
\hline Mayor de 24 meses & 41,5 & 16,8 & 9,4 & 58,6 & 30,9 & 33,8 & 29,1 & 35,1 \\
\hline \multicolumn{9}{|l|}{ Atención profesional en el parto } \\
\hline Sin atención & 45,3 & 26,0 & 25,2 & 66,4 & 35,1 & 49,3 & 40,5 & 52,6 \\
\hline Con atención & 23,0 & 14,5 & 9,7 & 50,0 & 21,8 & 28,3 & 22,7 & 20,4 \\
\hline
\end{tabular}

Fuente: elaboración propia sobre la base de las encuestas de demografía y salud (DHS). 


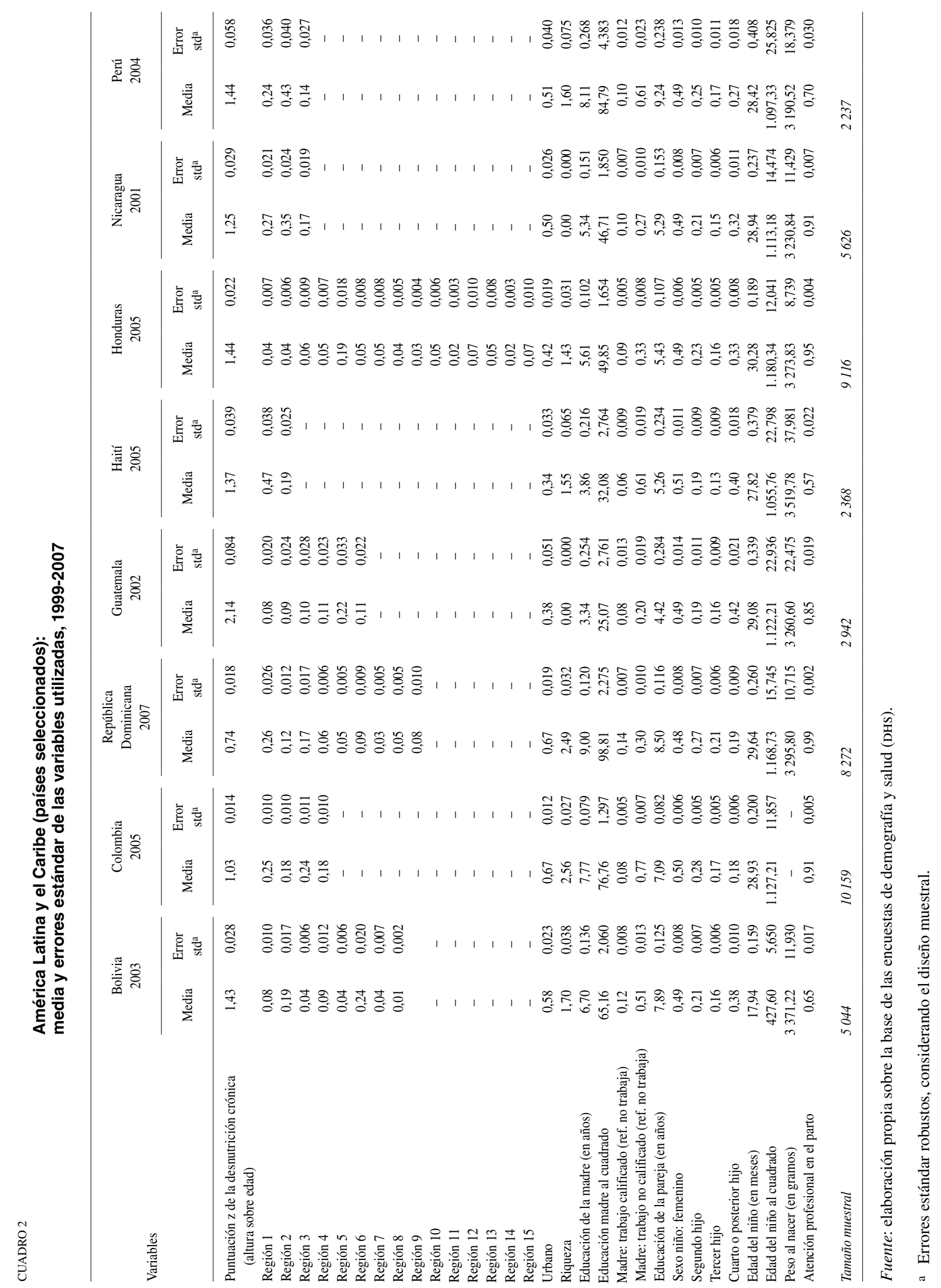




\section{III}

\section{La desigualdad en la desnutrición}

\section{y sus causas}

\section{Los determinantes de la desnutrición}

En el cuadro 3 se resumen los resultados de las regresiones lineales (mínimos cuadrados ordinarios (MCO)) para los ocho países considerados, junto con el valor de sus coeficientes $t$ (estimados utilizando errores estándar robustos).

Para todos los países, el modelo estimado tiene una buena capacidad explicativa, con $\mathrm{R}^{2}$ que van desde 0,10 para República Dominicana hasta 0,39 para el Perú. ${ }^{13}$ Incluso en los casos en que este indicador es comparativamente bajo, se obtuvieron altos valores del estadístico $\mathrm{F}$ (que mide de manera conjunta la significancia estadística de todos los coeficientes).

El coeficiente que acompaña al indicador de bienestar material, el índice de "riqueza" en este caso, es significativo y negativo para todos los países. ${ }^{14}$ No obstante, dada la naturaleza de este índice (de posesión de activos y servicios), no puede analizarse el significado singular del coeficiente. Es altamente probable que ciertas variables que lo componen, como la existencia de fuentes seguras de agua y eliminación de excretas, tengan un alto impacto individual en la desnutrición infantil, tal como se conoce también en la literatura (Smith, Ruel y Ndiaye, 2005).

La educación de la madre posee en todos los casos el signo esperado - una mayor educación materna tiene un efecto negativo sobre la desnutrición crónica-aunque solo es significativa en los casos de Bolivia, Colombia, Guatemala, Honduras y Nicaragua. El hecho de que, por ejemplo, para República Dominicana, dicho coeficiente no sea significativo no es sorprendente dado el alto nivel medio y la baja variabilidad de esta variable (véase el cuadro 2). En el caso de Haití, por ejemplo, si bien posee

\footnotetext{
${ }^{13} \mathrm{La}$ bondad del ajuste de un modelo es relativa al tipo de modelo que se esté estimando. Los ajustes obtenidos están dentro de los niveles que se observan en estudios similares a este (Wagstaff, Van Doorslaer y Watanabe, 2003; Harttgen y Misselhorn, 2006; Larrea y Kawachi, 2005).

14 Este resultado es consistente con lo reportado, entre otros, por Wagstaff, Van Doorslaer y Watanabe (2003); Valdivia (2004); Smith, Ruel y Ndiaye (2005); Larrea y Kawachi (2005); Chen, Eastwood y Yen (2007).
}

niveles de escolaridad promedio muy bajos, también es baja la variabilidad: poco más del $35 \%$ de las madres no poseen ninguna educación formal, mientras que el $40 \%$ que le sigue en educación se reparte prácticamente en partes iguales por año de escolaridad (es decir, que cada año de escolaridad tiene poco más del $6 \%$ de la muestra de madres). Esto hace que gran parte de la población (poco más del $75 \%$ ) tenga niveles muy bajos de escolaridad y se produzca una compresión en la distribución de esta variable (restándole poder explicativo).

El coeficiente que acompaña a la educación materna al cuadrado muestra rendimientos marginales decrecientes de la educación sobre la desnutrición infantil, lo que indicaría que, al menos en lo referido a la desnutrición, sería preferible disponer recursos para aumentar más la matrícula primaria que la secundaria. ${ }^{15}$ Además, en todos los casos el coeficiente que acompaña a la educación materna es superior al que acompaña a la educación de la pareja (que es solo significativo en el caso de Bolivia, Colombia y Honduras).

La edad y la edad al cuadrado de los niños son también en todos los casos altamente significativas y presentan signos esperables. A medida que la edad aumenta crece la incidencia de la desnutrición crónica, aunque este incremento ocurre a tasa decreciente. ${ }^{16}$ Asimismo, la desnutrición crónica tiende a afectar más a los varones que a las mujeres y este efecto es significativo en la mayoría de los casos (como en Marcoux, 2002). Por otra parte, el orden de nacimiento no parece ser importante (excepto en los casos de Colombia, Honduras y República Dominicana), a menos que consideremos órdenes de nacimiento altos ( $4^{\circ}$ o posterior). En este caso, los coeficientes son positivos y significativos (excepto en los casos de Bolivia y Haití).

La desnutrición crónica no parece tener un nivel diferente en el sector urbano respecto del sector rural,

\footnotetext{
${ }^{15}$ Este resultado es contrario al reportado por Larrea y Kawachi (2005) para el caso del Ecuador, aunque consistente con la mayor parte de la literatura consultada.

${ }^{16}$ Este resultado es consistente con el encontrado por Valdivia (2004), Larrea y Kawachi (2005) y Harttgen y Misselhorn (2006), aunque no por Wagstaff, Van Doorslaer y Watanabe (2003).
} 


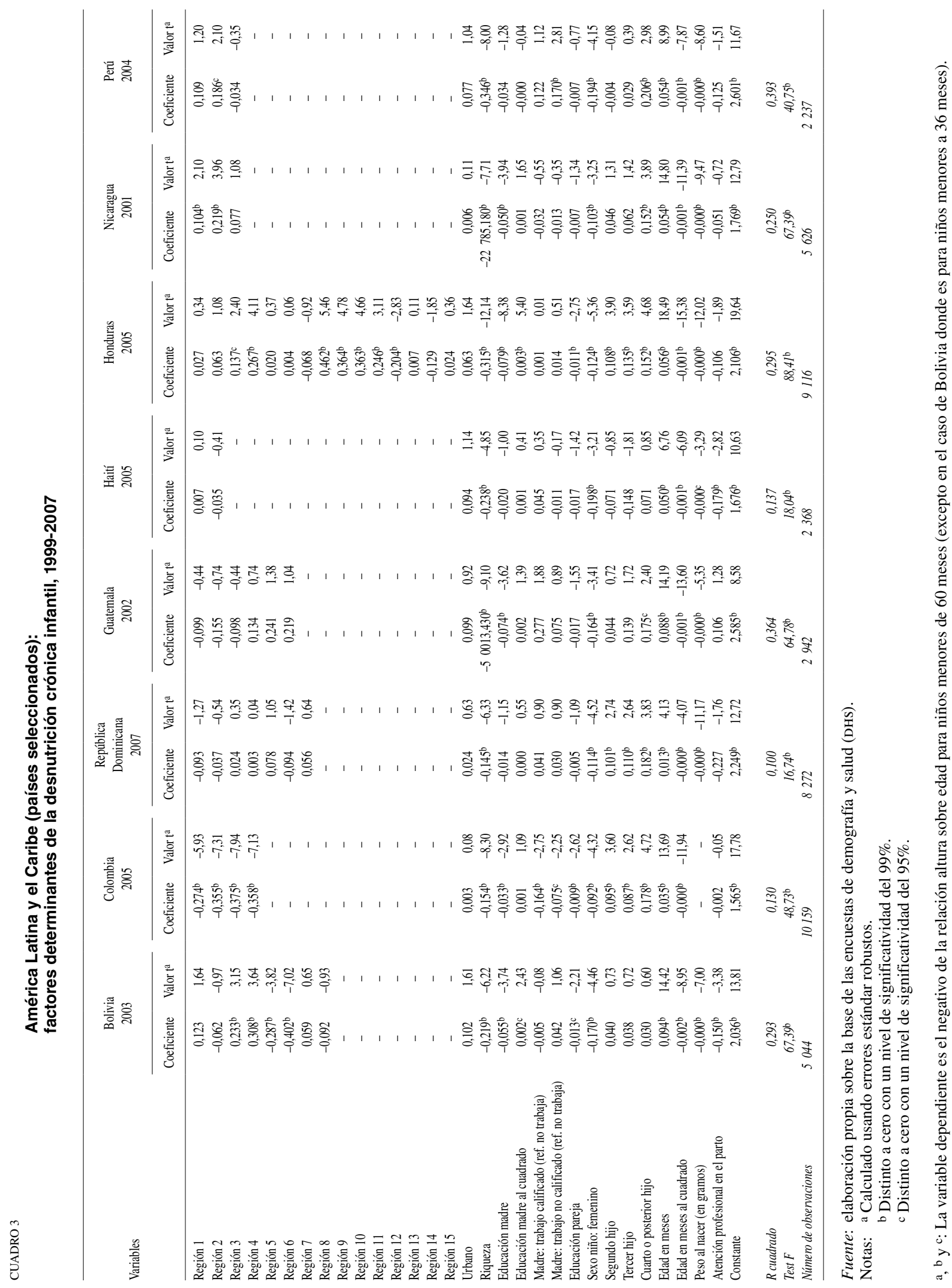


cuando se controla por el resto de las variables independientes (como en Smith, Ruel y Ndiaye, 2005 y Van de Poel y otros, 2007). Esto no implica que ella no sea más frecuente en un área geográfica específica —en el cuadro 1 se muestra que, de hecho, se presenta más a menudo en las zonas rurales-, sino que implica que la mayor o menor frecuencia obedece a la existencia concomitante de otras características, tales como el mayor o menor nivel de "riqueza" y de educación materna en estas áreas. En otras palabras, la dispersión observada en la desnutrición crónica no se explica, en la mayoría de los países, por las diferencias entre áreas geográficas, sino por las variables que dan cuenta de las diferencias en el interior de estas.

Es probable que la baja influencia de esta variable se vea también atenuada por los efectos fijos regionales, aunque estos no sean significativos en todos los casos y solo relevantes para determinados países. En Bolivia, por ejemplo, los niveles de desnutrición en Oruro y Potosí fueron significativamente mayores que los de La Paz, cuando se controla por el resto de las variables. En Colombia, todas las regiones tuvieron menores niveles promedio de desnutrición que el área de Bogotá (controlando por el resto de las variables). En el caso del Perú, la desnutrición fue mayor en la Sierra que en la región de Lima. Es probable que variables no consideradas como la "cultura", o más generalmente, factores idiosincrásicos, actúen por intermedio de las variables regionales en algunos países que poseen fuertes diferencias culturales/étnicas entre la región andina y el resto de las regiones (como Bolivia y el Perú).

Finalmente, el coeficiente que acompaña a la variable de acceso al sistema de salud (atención profesional del parto) solo es significativo en dos casos (Bolivia y Haití). ${ }^{17}$ No es casual que estos países sean los que registran menor acceso a este servicio en la muestra considerada. Mientras que en Bolivia y Haití solo el $65 \%$ y $57 \%$, respectivamente, de los partos son realizados por un profesional, en países como Colombia, Honduras, Nicaragua o República Dominicana, esta cobertura supera el 90\%. Debido a estas magnitudes - que muestran un acceso prácticamente universal a este servicio- esta variable deja de ser relevante para explicar el nivel promedio de desnutrición en la mayoría de los casos.

${ }^{17}$ Harttgen y Misselhorn (2006) encuentran que las variables de acceso a la infraestructura de salud tienen una importancia relativamente pequeña para explicar la desnutrición infantil (no así la mortalidad infantil).

\section{Causas de la desigualdad en la desnutrición}

Tal como se mencionó en la sección I, un aspecto clave en la situación presente $-\mathrm{y}$ futura ya que, como se explicó, este problema puede persistir fuertemente en el largo plazo- de la desnutrición crónica atañe a su distribución socioeconómica y a las causas de esta. Entonces, para el diseño de políticas es central comprender cómo afectan ciertos determinantes de la desnutrición crónica a su distribución.

En el cuadro 4 se aprecian las elasticidades (definidas en la ecuación (2)) y los índices de concentración (IC) de la desnutrición infantil y sus variables explicativas, todos ellos con el signo esperado. Aquellas variables que se concentran mayormente entre los hogares de mejor situación socioeconómica, como la "riqueza", la educación, el vivir en áreas urbanas o el acceso al sistema de salud, poseen IC positivos. Aquellas que se concentran, sobre todo entre los hogares de peor situación socioeconómica, como la desnutrición misma o un orden de nacimiento alto (cuarto o posterior), poseen un IC negativo. Otras variables que no poseen un patrón establecido de concentración socioeconómica, como el sexo del niño o su edad, pueden tener IC positivos o negativos, pero en cualquier caso cercanos a cero - mostrando justamente la inexistencia de un patrón claro de concentración socioeconómica-.

Además, los IC de la desnutrición infantil —que se descomponen según las ecuaciones (2) y (3) — muestran diferentes realidades para el grupo de países analizados. Colombia y Haití, por ejemplo, son los países con IC más cercanos a cero $\mathrm{y}$, por ende, los que tienen una menor desigualdad socioeconómica en desnutrición. No obstante, esto no implica que sus realidades sean similares, ya que mientras el primero registra una prevalencia nacional de la desnutrición del 15,6\% (esto es, el 15,6\% de los niños menores de 60 meses presentan algún grado de desnutrición crónica), en Haití alcanza al $27,6 \%$. Entonces, la menor prevalencia nacional y la relativamente baja desigualdad socioeconómica en su distribución hacen que la situación en Colombia sea "preferible" a la registrada en Haití.

En el otro extremo se encuentran Guatemala, Nicaragua y el Perú con Ic para la desnutrición infantil iguales a $-0,191,-0,196$ y $-0,231$, respectivamente. En el caso particular de Guatemala, el IC relativamente alto se asocia también con la mayor prevalencia a nivel nacional de toda la muestra, lo que señalaría la existencia de un patrón de "privación masiva" con una alta concentración en los hogares con peores condiciones materiales. 


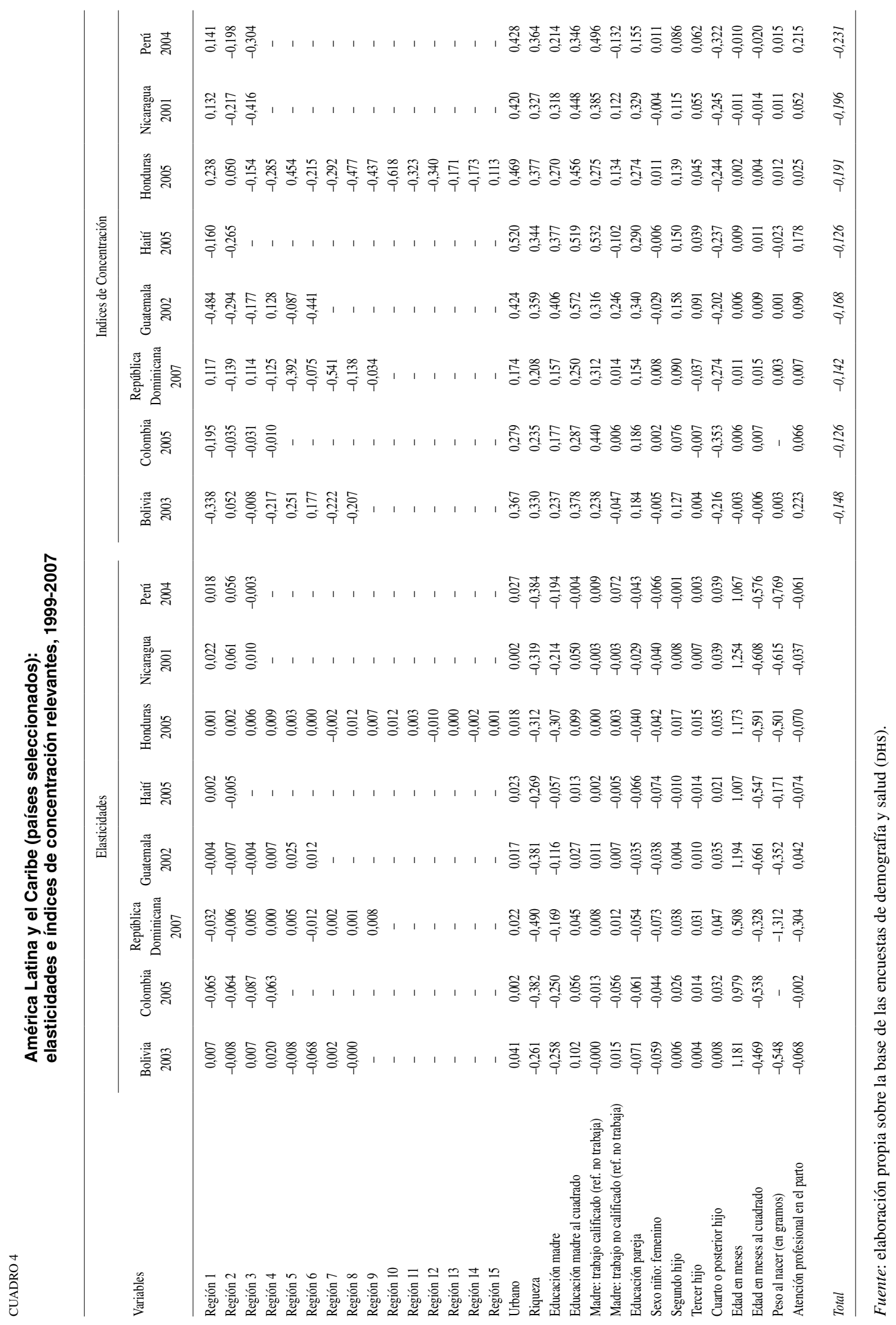


A su vez, en el cuadro 5 se presenta la descomposición del IC de la desnutrición según sus causas (de acuerdo con la ecuación (3)).

En todos los casos, la "riqueza" es lejos la variable más importante para explicar la desigualdad socioeconómica en la desnutrición crónica infantil. La contribución marginal de esta variable (manteniendo el resto de los factores constantes) oscila entre un 53\% en Nicaragua y un $81 \%$ en Guatemala. Incluso en países con una prevalencia relativamente baja de la desnutrición crónica, como es el caso de República Dominicana, la contribución marginal de la "riqueza" rebasa el 71\%. Esto quiere decir que, en el caso de República Dominicana, si desaparecieran las disparidades en la distribución de la "riqueza" (manteniendo constantes todos los otros factores), la desigualdad en la desnutrición infantil se reduciría en un $71 \%$.

Esta importante contribución marginal se explica por altas elasticidades-"riqueza" de la desnutrición (véase el cuadro 4) y, sobre todo, por altos niveles de concentración de la "riqueza" (como muestran los respectivos IC en el cuadro 4). No es una novedad en la literatura que los países de la región poseen altos niveles de desigualdad socioeconómica (De Ferranti y otros, 2004), pero es novedoso el resultado de que esta alta desigualdad tenga un impacto de tal magnitud en un problema sanitario como la desnutrición infantil. En este sentido, disminuir la desnutrición — sobre la base de una reducción en su desigualdad socioeconómica—implica no solo adoptar políticas sanitarias adecuadas (que, por

CUADRO 5

\begin{abstract}
América Latina y el Caribe (países seleccionados): factores determinantes de la desigualdad en la desnutrición crónica infantil, 1999-2007 (En porcentajes)
\end{abstract}

\begin{tabular}{|c|c|c|c|c|c|c|c|c|}
\hline Variables & $\begin{array}{c}\text { Bolivia } \\
2003\end{array}$ & $\begin{array}{c}\text { Colombia } \\
2005\end{array}$ & $\begin{array}{c}\text { República } \\
\text { Dominicana } \\
2007\end{array}$ & $\begin{array}{c}\text { Guatemala } \\
2002\end{array}$ & $\begin{array}{l}\text { Haití } \\
2005\end{array}$ & $\begin{array}{l}\text { Honduras } \\
2005\end{array}$ & $\begin{array}{c}\text { Nicaragua } \\
2001\end{array}$ & $\begin{array}{l}\text { Perú } \\
2004\end{array}$ \\
\hline Región 1 & 1,51 & $-10,13$ & 2,67 & $-1,09$ & 0,30 & $-0,10$ & $-1,50$ & $-1,11$ \\
\hline Región 2 & 0,29 & $-1,75$ & $-0,58$ & $-1,16$ & $-1,04$ & $-0,04$ & 6,73 & 4,74 \\
\hline Región 3 & 0,04 & $-2,13$ & $-0,44$ & $-0,47$ & - & 0,48 & 2,20 & $-0,45$ \\
\hline Región 4 & 2,96 & $-0,51$ & 0,02 & $-0,51$ & - & 1,36 & - & - \\
\hline Región 5 & 1,39 & - & 1,33 & 1,28 & - & $-0,61$ & - & - \\
\hline Región 6 & 8,13 & - & $-0,62$ & 3,04 & - & 0,02 & - & - \\
\hline Región 7 & 0,25 & - & 0,84 & - & - & $-0,37$ & - & - \\
\hline Región 8 & $-0,06$ & - & 0,09 & - & - & 3,04 & - & - \\
\hline Región 9 & & - & 0,20 & - & - & 1,66 & - & - \\
\hline Región 10 & & - & - & - & - & 3,82 & - & - \\
\hline Región 11 & & - & - & - & - & 0,54 & - & - \\
\hline Región 12 & & - & - & - & - & $-1,79$ & - & - \\
\hline Región 13 & & - & - & - & - & 0,02 & - & - \\
\hline Región 14 & & - & - & - & - & $-0,18$ & - & - \\
\hline Región 15 & & - & - & - & - & $-0,07$ & - & - \\
\hline Urbano & $-10,24$ & $-0,40$ & $-2,63$ & $-4,39$ & $-9,60$ & $-4,51$ & $-0,50$ & $-5,07$ \\
\hline Riqueza & 58,27 & 71,31 & 71,41 & 81,80 & 73,62 & 61,59 & 53,22 & 60,39 \\
\hline Educación madre & 41,33 & 35,32 & 18,65 & 28,21 & 17,09 & 43,43 & 34,75 & 17,95 \\
\hline Educación madre al cuadrado & $-25,91$ & $-12,73$ & $-7,95$ & $-9,35$ & $-5,33$ & $-23,67$ & $-11,36$ & 0,53 \\
\hline Madre: trabajo calificado (ref. no trabaja) & 0,07 & 4,53 & $-1,76$ & $-2,03$ & $-0,79$ & $-0,01$ & 0,52 & $-1,88$ \\
\hline Madre: trabajo no calificado (ref. no trabaja) & 0,48 & 0,29 & $-0,12$ & $-1,01$ & $-0,41$ & $-0,23$ & 0,17 & 4,13 \\
\hline Educación pareja & 8,86 & 8,99 & 5,85 & 7,14 & 15,20 & 5,70 & 4,83 & 2,88 \\
\hline Sexo niño: femenino & $-0,21$ & 0,05 & 0,41 & $-0,66$ & $-0,35$ & 0,24 & $-0,08$ & 0,30 \\
\hline Segundo hijo & $-0,51$ & $-1,58$ & $-2,38$ & $-0,38$ & 1,21 & $-1,26$ & $-0,46$ & 0,03 \\
\hline Tercer hijo & $-0,01$ & 0,08 & 0,83 & $-0,55$ & 0,45 & $-0,35$ & $-0,21$ & $-0,09$ \\
\hline Cuarto o posterior hijo & 1,18 & 8,88 & 9,06 & 4,18 & 3,89 & 4,43 & 4,92 & 5,45 \\
\hline Edad en meses & 2,17 & $-4,86$ & $-3,87$ & $-4,30$ & $-6,85$ & $-0,97$ & 6,81 & 4,72 \\
\hline Edad en meses al cuadrado & $-1,98$ & 2,86 & 3,45 & 3,42 & 4,74 & 1,27 & $-4,21$ & $-4,86$ \\
\hline Peso al nacer (en gramos) & 1,15 & - & 2,68 & 0,29 & $-3,06$ & 3,05 & 3,35 & 5,13 \\
\hline Atención profesional en el parto & 10,27 & 0,10 & 1,48 & $-2,25$ & 10,49 & 0,92 & 0,97 & 5,68 \\
\hline Descomposición total explicada & 99,41 & 98,30 & 98,63 & 101,23 & 99,55 & 97,42 & 100,16 & 98,45 \\
\hline Proporción no explicada & 0,59 & 1,70 & 1,37 & $-1,23$ & 0,45 & 2,58 & $-0,16$ & 1,55 \\
\hline Total & 100,00 & 100,00 & 100,00 & 100,00 & 100,00 & 100,00 & 100,00 & 100,00 \\
\hline
\end{tabular}

Fuente: elaboración propia sobre la base de las encuestas de demografía y salud (DHS). 
ejemplo, detecten rápidamente casos de desnutrición infantil y prevean mecanismos correctivos), sino atacar desigualdades materiales básicas que condicionarían a la desnutrición, más allá de tales políticas sanitarias. El abanico de políticas que debieran ser aplicables a este problema excedería largamente a las políticas de salud y se extendería entonces, por ejemplo, a las políticas habitacionales, laborales, de ingresos y macroeconómicas a objeto de asegurar un entorno de estabilidad económica a los hogares de menores recursos.

Las variables educativas —educación de la madre y de la pareja - son las segundas grandes contribuyentes que explican las desigualdades en la desnutrición crónica infantil y, dentro de estas, es la educación materna la que posee un mayor impacto marginal. Como se mencionó en la sección anterior, cuanto mayor es la educación materna (en años de escolaridad), menor tiende a ser la desnutrición infantil. Debido a que la educación se encuentra relativamente concentrada entre los hogares más "ricos" (su IC es fuertemente positivo, en todos los casos), ella contribuye positivamente a explicar la desigualdad en la desnutrición. Pero este efecto pro-desigualdad de la educación se ve atenuado por la existencia de otro, no lineal, entre la educación materna y la desnutrición: el efecto de esta variable en la desnutrición tiende a perderse conforme aumentan los años de escolaridad (y estos crecen en los hogares relativamente más prósperos), por lo que el resultado final de esta variable es menor al que se tendría si se considerara solo la educación materna en años. El resultado final (considerando ambos efectos) va desde un $10 \%$ en el caso de República Dominicana a un $23 \%$ en Colombia y Nicaragua. ${ }^{18}$

Las variables biomédicas (sexo, edad, orden de nacimiento, peso al nacer) no tienen, como es de esperar, gran importancia a la hora de explicar la desigualdad en la desnutrición crónica infantil, aunque algunas de ellas sean relevantes para explicar su nivel. El sexo de los niños, por ejemplo, no tiene un patrón de distribución socioeconómico definido (por ejemplo, las niñas no son más o menos frecuentes en los hogares "pobres" que en los "ricos") y, por ello, no tiene en este contexto ningún poder explicativo. Las restantes variables tienen por separado un bajo efecto (las de orden de nacimiento son las que poseen mayor poder explicativo, sobre todo si se trata de órdenes altos de nacimiento), pero tomadas en conjunto nunca llegan a explicar más del 10\% de la desigualdad en la desnutrición crónica.

\footnotetext{
${ }^{18}$ Esto implicaría que, por ejemplo, en el caso de Nicaragua, si todas las madres hubieran tenido el mismo nivel educativo (igual a la media nacional), la desigualdad en la desnutrición crónica hubiera sido, ceteris paribus, inferior en un $23 \%$ a la registrada.
}

Las variables geográfico-regionales tampoco tienen un patrón general definido, sino que dependen de cada país. Mientras que en Guatemala, Honduras o República Dominicana ni las variables regionales ni la de área (urbana/rural) contribuyen a explicar significativamente la desigualdad en la distribución de la desnutrición crónica (verbigracia, los niños desnutridos no se concentran en una región o área específica cuando se mantienen constantes las otras variables consideradas), en países como Colombia la contribución es considerable. Por ejemplo, la región 1 (Atlántica) tiene un nivel sustancialmente menor de desnutrición crónica que la región de referencia (Bogotá) y posee una proporción importante de hogares pobres. Estos dos factores conjuntamente redundan en que su contribución a la desigualdad en la desnutrición sea negativa (es decir que, ceteris paribus, la menor desnutrición en la región Atlántica se traduciría en una menor desigualdad en la desnutrición crónica nacional). Por otra parte, en Bolivia los hogares urbanos tienen mejores condiciones materiales que los rurales (el IC de área para Bolivia es altamente positivo y muestra que los hogares urbanos se concentran entre los hogares más "ricos"), aunque los niños de aquellos hogares tienen peores indicadores nutricionales que el promedio (cuando se mantienen constantes las otras variables explicativas). Esto hace que el efecto de esta variable en la desigualdad en la desnutrición crónica infantil sea también negativo (10\%).

Finalmente, la variable de acceso al sistema de salud (atención profesional del parto) tiene un comportamiento dispar entre los países. En aquellos donde la cobertura es relativamente baja - por ejemplo, Bolivia, Haití y el Perú, donde solo el $65 \%, 57 \%$ y $70 \%$ de los partos, respectivamente, son realizados por profesionales-, la contribución de este factor para explicar la desigualdad en la desnutrición crónica va desde un $6 \%$ a un $10 \%$. Por otra parte, en aquellos países donde el servicio de atención profesional del parto - y el acceso al sistema de salud, en general - tiene una cobertura importante (como por ejemplo, Colombia o República Dominicana) el efecto es naturalmente pequeño debido a que las desigualdades socioeconómicas tienden a desaparecer en su distribución, lo que también hace desaparecer el efecto explicativo en la desnutrición promedio al eliminar la varianza de la variable. No obstante, el hecho de que en estos países la gran mayoría de la población acceda a, por ejemplo, atención profesional en el parto, no implica que no persistan diferencias socioeconómicas en la calidad del servicio que reciben. Lamentablemente no existen instrumentos en las encuestas DHS para incorporar esta dimensión. 


\section{IV}

\section{Conclusiones}

Existen numerosas causas que determinan el nivel de la desnutrición crónica infantil en un país y esas causas, por lo general, poseen un complejo entramado de interrelaciones. Las variables geográficas o de área pueden parecer importantes a la hora de explicar el nivel y la desigualdad de la desnutrición, pero una vez que son consideradas dentro de modelos amplios con factores explicativos adicionales, pierden importancia relativa. En este trabajo se muestra qué variables son las más relevantes cuando se quiere explicar el promedio de la desnutrición y su distribución socioeconómica. En los países estudiados, donde las desigualdades socioeconómicas son importantes, una disminución permanente de la desnutrición solo puede alcanzarse si se atacan sus condicionantes socioeconómicas. En tal sentido, las políticas públicas necesarias para disminuir la incidencia de este problema deben ser amplias: si bien la desnutrición es un problema sanitario - con importantes consecuencias económicas- las políticas para su reducción deben ser no solo de salud sino de ingresos, educativas, de vivienda, y otras.

Dentro de estas condicionantes socioeconómicas, la "riqueza" (tal como se mide en las DHs) es la mayor contribuyente para explicar las desigualdades en la desnutrición infantil. El indicador de "riqueza" utilizado no mide ingresos/gastos de las familias, sino condiciones de habitabilidad del hogar, tenencia de activos y servicios disponibles. Entonces, la mejor distribución de la "riqueza" — que como se muestra aquí tendría una importante efecto en la distribución y el nivel de la desnutrición - no implica necesariamente quitarle recursos a un grupo para dárselos a otro, sino, por ejemplo, mejorar el acceso a agua potable y saneamiento adecuado para los hogares que no lo tienen. Naturalmente, este tipo de políticas pueden tener potencialmente un efecto redistributivo dependiendo de la progresividad de la estructura tributaria.

El nivel educacional de los padres, pero especialmente de la madre, es otra variable que influye decisivamente en el nivel y la distribución de la desnutrición crónica. Pero para que las mejoras en la educación tengan un efecto pleno en la desnutrición deben beneficiar principalmente a los hogares más "pobres" y concentrarse primeramente en el nivel educativo básico. Países como Bolivia, Guatemala, Haití, Honduras y Nicaragua se encuentran todavía lejos de lograr la escolarización primaria universal (aunque algunos han avanzado en ese sentido) y tienen camino por recorrer, lo que seguramente implicará una mejora en indicadores como la desnutrición crónica infantil. De manera similar, las políticas tendientes a mejorar la inserción femenina en el mercado laboral, como por ejemplo las que brindan cuidado de niños para madres que trabajan fuera del hogar, pueden también tener un efecto positivo en la desnutrición infantil, tanto mayor cuanto más focalizadas estén las políticas en madres de hogares pobres o con ingresos insuficientes.

Factores geográficos, culturales, étnicos e idiosincrásicos juegan algún rol explicativo, pero, aparentemente, a partir de su relación con la distribución de las variables socioeconómicas, principalmente la "riqueza" y la educación materna. Esto no implica que no tengan importancia a la hora de diseñar políticas sectoriales o que las políticas públicas deban soslayarlos. Lo que este hecho señala es que las políticas públicas para disminuir la desnutrición crónica infantil deberían diseñarse no sobre la base de la localización del hogar, sino de sus características socioeconómicas (bienestar material, educación de la madre y su pareja, entre otros). Solo mediante la reducción de estas desigualdades los países de la región pueden aspirar a dejar en el pasado la desnutrición infantil y sus terribles consecuencias. 
ANEXO

CUADRO A

América Latina y el Caribe (países seleccionados): agrupación regional de departamentos o provincias

\begin{tabular}{ll}
\hline País & Región \\
\hline Bolivia & \\
& La Paz (Región 0) \\
& Chuquisaca (Región 1) \\
& Cochabamba (Región 2) \\
& Oruro (Región 3) \\
& Potosí (Región 4) \\
& Tarija (Región 5) \\
& Santa Cruz (Región 6) \\
& Beni (Región 7) \\
& Pando (Región 8) \\
Colombia & Bogotá (Región 0) \\
& Atlántica (Región 1) \\
& Oriental (Región 2) \\
República Dominicana & Central (Región 3) \\
& Pacífica (Región 4) \\
& Distrito Nacional (Región 0) \\
& 0 (Región 1)
\end{tabular}

I (Región 2)

II (Región 3)

III (Región 4)

IV (Región 5)

V (Región 6)

VI (Región 7)

VII (Región 8)

VIII (Región 9) Monte Plata

Azua

Peravia

San Cristóbal

San José de Ocoa

Espaillat

Puerto Plata

Santiago

Duarte

Hermanas Mirabal

María Trinidad Sánchez

Samaná

Bahoruco

Barahona

Independencia

Pedernales

El Seibo

La Altagracia

La Romana

Hato Mayor

San Pedro de Macorís

Elías Piña

San Juan

Dajabón

Monte Cristi

Santiago Rodríguez

Valverde

La Vega

Monseñor Nouel

Sánchez Ramírez

Guatemala

Metropolitana (Región 0)

Norte (Región 1)

Noreste (Región 2)

Sureste (Región 3 )

Central (Región 4)

Suroeste (Región 5 )

Noroeste (Región 6)

Haití

Area Metropolitana (Región 0)

Norte (Región 1)

Norte

Noreste 
(continuación)

\begin{tabular}{ll}
\hline País & Región \\
\hline Haití & \\
& \\
& Sur (Región 2) \\
& \\
Honduras & \\
& \\
& Francisco Morazán (Región 0) \\
& Atlántida (Región 1) \\
& Colón (Región 2) \\
& Comayagua (Región 3) \\
& Copán (Región 4) \\
& Cortés (Región 5) \\
& Choluteca (Región 6) \\
& El Paraíso (Región 7) \\
& Intibucá (Región 8) \\
& La Paz (Región 9) \\
& Lempira (Región 10) \\
& Ocotepeque (Región 11) \\
& Olancho (Región 12) \\
Nicaragua & Santa Bárbara (Región 13) \\
& Valle (Región 14) \\
& Yoro (Región 15) \\
&
\end{tabular}

Managua (Región 0)

Pacífico (Región 1)

Chinandega

León

Masaya

Granada

Carazo

Central norte (Región 2)

Rivas

Boaco

Chontales

Jinotega

Matagalpa

Estelí

Madriz

Atlántico (Región 3)

Nueva Segovia

Río San Juan

Región Atlántico Norte (RAAN)

Región Atlántico Sur (RAAS)

Perú

Lima (Región 0)
Costa (Región 1)

$\begin{array}{ll} & \text { Callao } \\ \text { Ica } & \text { La Libertad } \\ & \text { Lambayeque } \\ \text { Moquegua } & \text { Piura } \\ \text { Tacna } & \text { Tumbes } \\ \text { Sierra (Región 2) } & \text { Áncash } \\ & \text { Apurímac } \\ & \text { Arequipa } \\ & \text { Ayacucho } \\ & \text { Cajamarca } \\ & \text { Cusco } \\ \text { Huncavelica } & \text { Huánuco } \\ \text { Selva (Región 3) } & \text { Junín } \\ & \text { Pasco } \\ \text { Puno } \\ \\ \\ \end{array}$

Fuente: elaboración propia sobre la base de las encuestas de demografía y salud (DHS). 
Bibliografía

Armar-Klemesu, M. y otros (2000), "Poor maternal schooling is the main constraint to good child care practices in Accra", Journal of Nutrition, vol. 130, $\mathrm{N}^{\circ}$ 6, Bethesda, Maryland, American Society for Nutrition.

Black, R. y otros (2008), "Maternal and child undernutrition: global and regional exposures and health consequences", The Lancet, vol. 371, N 9608, Amsterdam, Elsevier.

CEPAL (Comisión Económica para América Latina y el Caribe) (2008), Objetivos de desarrollo del Milenio. La progresión hacia el derecho a la salud en América Latina y el Caribe (LC/G.2364), Santiago de Chile.

Cleland, J., y J. Van Ginneken (1988), "Maternal education and child survival in developing countries: The search for pathways of influence", Social Science \& Medicine, vol. 27, № 12 , Amsterdam, Elsevier.

Chen, Z., D. Eastwood y S. Yen (2007), “A decade's story of childhood malnutrition inequality in China: Where you live does matter", China Economic Review, vol. 18, No 2, Amsterdam, Elsevier.

De Ferranti, D. y otros (2004), Inequality in Latin America: Breaking with History?, Washington, D.C., Banco Mundial.

Drèze, J. y A. Sen (1989), Hunger and Public Action, Oxford, Clarendon Press.

Filmer, D. y L. Pritchett (2001), "Estimating wealth effects without expenditure data-or tears: an application to educational enrolments in states of India", Demography, vol. 38, No 1, Silver Spring, Population Association of America.

Grossman, M. (1972), "On the concept of health capital and the demand for health", Journal of Political Economy, vol. 80, $\mathrm{N}^{\mathrm{o}}$ 2, Chicago, University of Chicago Press.

Gwatkin, D. y otros (2007), Socio-Economic Differences in Health, Nutrition, and Population within Developing Countries. An Overview, Washington, D.C., Banco Mundial [en línea] http:// go.worldbank.org/XJK7WKSE40

Harttgen, K. y M. Misselhorn (2006), "A multilevel approach to explain child mortality and undernutrition in South Asia and Sub-Saharan Africa" [en línea] http://opus.zbw-kiel.de/volltexte/2006/4743/ pdf/Misselhorn.pdf

Hosseinpoor, A. y otros (2006), "Decomposing socioeconomic inequality in infant mortality in Iran”, International Journal of Epidemiology, vol. 35, No 5, Oxford, Oxford University Press.

Jewell, T., P. Triunfo y R. Aguirre (2007), "El peso al nacer de los niños de la principal maternidad de Uruguay: 1995 a 2004", Desarrollo y sociedad, Bogotá, D.C., Centro de Estudios sobre Desarrollo Económico, Universidad de los Andes.

Kakwani, N., A. Wagstaff y E. van Doorslaer (1997), "Socioeconomic inequalities in health: Measurement, computation, and statistical inference", Journal of Econometrics, vol. 77, No 1, Amsterdam, Elsevier.

Larrea, C. (2002), "Desigualdad social, salud materno-infantil y nutrición en ocho países de América Latina: Análisis comparativo de las encuestas DHS III", Washington, D.C., Organización Panamericana de la Salud [en línea] http://www.paho.org/Spanish/HPP/HPN/ larrea-encuestaDHS.htm

Larrea, C. y W. Freire (2002), "Social inequality and child malnutrition in four Andean countries", Revista panamericana de salud pública, vol. 11, N N $^{\circ}$ 5-6, Washington, D.C., Organización Panamericana de la Salud.

Larrea, C. e I. Kawachi (2005), "Does economic inequality affect child malnutrition? The case of Ecuador", Social Science and Medicine, vol. 60, Amsterdam, Elsevier.

Lavy, V. y otros (1996), "Quality of health care, survival and health outcomes in Ghana", Journal of Health Economics, vol. 15, $\mathrm{N}^{\circ} 3$, Amsterdam, Elsevier.

Marcoux, A. (2002), "Sex differentials in undernutrition: A look at survey evidence", Population and Development Review, vol. 28, $\mathrm{N}^{\mathrm{o}} 2$, Nueva York, The Population Council.
Martínez, R. (coord.) (2005), "Hambre y desigualdad en los países andinos. La desnutrición y la vulnerabilidad alimentaria en Bolivia, Colombia, Ecuador y Perú”, serie Políticas sociales, No 112 (LC/L.2400-P), Santiago de Chile, Comisión Económica para América Latina y el Caribe (CEPAL). Publicación de las Naciones Unidas, $N^{\circ}$ de venta: S.05.II.G.147.

Martínez, R. y A. Fernández (2006), "Modelo de análisis del impacto social y económico de la desnutrición infantil en América Latina", serie Manuales, $\mathrm{N}^{\circ} .52$ (LC/L.2650-P), Santiago de Chile, Comisión Económica para América Latina y el Caribe (CEPAL). Publicación de las Naciones Unidas, $\mathrm{N}^{\circ}$ de venta: S.06.II.G. 175 .

OMs (Organización Mundial de la Salud) (2008), Subsanar las desigualdades en una generación, Ginebra, Comisión sobre Determinantes Sociales de la Salud.

(2006), wHO Child Growth Standards: Length/height-forage, Weight-for-age, Weight-for-length, Weight-for-height and Body Mass Index-for-age: Methods and Development, Ginebra.

(2001), Macroeconomics and Health: Investing in Health for Economic Development, Ginebra, Comisión sobre Macroeconomía y Salud.

Paraje, G. (2008), "Evolución de la desnutrición crónica infantil y su distribución socioeconómica en siete países de América Latina y el Caribe", serie Políticas sociales, No 140 (LC/L.2878-P), Santiago de Chile, Comisión Económica para América Latina y el Caribe (CEPAL). Publicación de las Naciones Unidas, $\mathrm{N}^{\circ}$ de venta: S.08.II.G.17.

Pelletier, D. y otros (1995), "The effect of malnutrition on child mortality in developing countries", Bulletin of the World Health Organization, vol. 73, Washington, D.C., Organización Mundial de la Salud.

Rutstein, S. y K. Johnson (2004), “The DHS Wealth Index”, DHS Comparative Reports, $\mathrm{N}^{\circ}$ 6, Calverton, ORC Macro.

Schultz, T. (1984), "Studying the impact of household economic and community variables on child mortality", Population and Development Review, vol. 10, Nueva York, The Population Council.

Smith, L., M. Ruel y A. Ndiaye (2005), "Why is child malnutrition lower in urban than in rural areas? Evidence from 36 developing countries", World Development, vol. 33, N 8, Amsterdam, Elsevier.

Thomas, D., V. Lavy y J. Strauss (1996), "Public policy and anthropometric outcomes in the Côte d'Ivoire", Journal of Public Economics, vol. 61, No 2, Amsterdam, Elsevier.

Valdivia, M. (2004), "Poverty, health infrastructure and the nutrition of Peruvian children", Economics and Human Biology, vol. 2, $\mathrm{N}^{\mathrm{o}} 3$, Amsterdam, Elsevier.

Van de Poel, E., O. O’Donnell y E. van Doorslaer (2007), "Are urban children really healthier? Evidence from 47 developing countries", Social Science and Medicine, vol. 65, Amsterdam, Elsevier.

Van de Poel, E. y otros (2007), "Malnutrition and socioeconomic gaps in malnutrition in Ghana", International Journal for Equity in Health, vol. 6, Londres, International Society for Equity in Health.

Victora, C. y otros (2008), "Maternal and child undernutrition: consequences for adult health and human capital", The Lancet, vol. 371, No 9609, Amsterdam, Elsevier.

Wagstaff, A., P. Paci y E. van Doorslaer (1991), "On the measurement of inequalities in health", Social Science and Medicine, vol. 33, Amsterdam, Elsevier.

Wagstaff, A., E. van Doorslaer y N. Watanabe (2003), “On decomposing the causes of health sector inequalities with an application to malnutrition inequalities in Vietnam", Journal of Econometrics, vol. 112, No 1 , Amsterdam, Elsevier. 